\title{
First Simulations of Axion Minicluster Halos
}

\author{
Benedikt Eggemeier ${ }^{1,{ }^{*}}$ Javier Redondo ${ }^{2,3, \dagger}$ Klaus Dolag, ${ }^{4,5}$ Jens C. Niemeyer, ${ }^{1,6}$ and Alejandro Vaquero ${ }^{7}$ \\ ${ }^{1}$ Institut für Astrophysik, Georg-August-Universität Göttingen, D-37077 Göttingen, Germany \\ ${ }^{2}$ CAPA \& Departamento de Física Teórica, Universidad de Zaragoza, 50009 Zaragoza, Spain \\ ${ }^{3}$ Max-Planck-Institut für Physik, D-80805 München, Germany \\ ${ }^{4}$ Max-Planck-Institut für Astrophysik, D-85741 Garching, Germany \\ ${ }^{5}$ University Observatory Munich, D-81679 München, Germany \\ ${ }^{6}$ Department of Physics, University of Auckland, Private Bag 92019, Auckland, New Zealand \\ ${ }^{7}$ Department of Physics and Astronomy, University of Utah, Salt Lake City, Utah 84112, USA
}

(Received 22 November 2019; revised 4 May 2020; accepted 29 June 2020; published 20 July 2020)

\begin{abstract}
We study the gravitational collapse of axion dark matter fluctuations in the postinflationary scenario, socalled axion miniclusters, with $\mathrm{N}$-body simulations. Largely confirming theoretical expectations, overdensities begin to collapse in the radiation-dominated epoch and form an early distribution of miniclusters with masses up to $10^{-12} M_{\odot}$. After matter-radiation equality, ongoing mergers give rise to a steep powerlaw distribution of minicluster halo masses. The density profiles of well-resolved halos are Navarro-FrenkWhite-like to good approximation. The fraction of axion dark matter in these bound structures is $\sim 0.75$ at redshift $z=100$.
\end{abstract}

DOI: 10.1103/PhysRevLett.125.041301

The QCD axion is a hypothetical particle predicted in the Peccei-Quinn (PQ) mechanism for solving the strong $C P$ problem and is considered one of the best motivated dark matter (DM) candidates [1-8]. In the so-called postinflation scenario, the axion field takes initial conditions after a phase transition happening after cosmic inflation, and its resulting DM density distribution has large fluctuations on subparsec comoving scales. Their gravitational collapse results in the formation of so-called axion miniclusters (MCs) with characteristic masses and radii of order $M_{\mathrm{mc}} \sim$ $10^{-12} M_{\odot}$ and $R_{\mathrm{mc}} \sim 10^{12} \mathrm{~cm}$ [9-12], a range in which they could be detected in femto-, pico- [12], and microlensing surveys [13]. Note that the estimates depend strongly on the cosmological assumptions before big bang nucleosynthesis $[14,15]$.) Moreover, the clumping of DM axions in bound objects has a direct implication in the direct detection at terrestrial experiments [16-18] and could have an impact in indirect detection [19,20] (see also [21]. Thus, quantitative predictions for the distribution of axions and the properties of MCs in this scenario are important.

The evolution of axion DM can roughly be split into three separate stages. The first encompasses the evolution of the axion field from PQ symmetry breaking until after the QCD phase transition when the axion mass has reached its low-temperature value. This early-universe epoch occurs well before the onset of gravitational instability and is governed by the formation and decay of topological defects and nonlinear field dynamics. This has recently been investigated, with special focus on MC formation, by means of large lattice simulations [22,23]. During the second stage, gravity takes over as the dominant force while scalar field gradients can be neglected on the scales of density perturbations, allowing their description with $N$-body methods for collisionless fluids [24]. Semianalytic tools for structure formation can be employed to predict the properties of minicluster halos (MCHs) such as the minicluster halo mass function (MC-HMF) [13]. Finally, MCHs evolve into large-scale DM halos and become the sites of galaxy formation in the third epoch. Tidal disruption of $\mathrm{MCHs}$ and the formation of axion streams are of particular importance during this final stage in order to predict the statistics of axion clumping at the present time [16,25].

This Letter reports the first results from large $N$-body simulations addressing the second stage of this process, the formation of axion MCHs by gravitational collapse of primordial axion density perturbations. In particular, we discuss the evolution of the MC-HMF, the fraction of axions bound into $\mathrm{MCHs}$, and the $\mathrm{MCH}$ density profile. More detailed statistics will be presented in a follow-up publication.

Simulations of axion density perturbations.-We start from initial conditions produced by early-universe simulations using the methods described in Ref. [22]. The frozen density distribution resulting from the evolution of the axion field at redshift $z \simeq 10^{6}$ was converted to $1024^{3}$ particles in a box with comoving side length $L=0.864 \mathrm{pc}$ and periodic boundary conditions. The length corresponds to $24 L_{1}$ where $L_{1}=2\left[1+z\left(t_{1}\right)\right] t_{1}$ is the comoving coherence length of the axion field at the time $t_{1}$ when its mass starts to dominate its dynamics $\left\{m_{A}\left(t_{1}\right) t_{1}=1 / 2\right.$, see Supplemental Material (SM) [26]\}. For simplicity, we assume that axions account for the total amount of DM. 

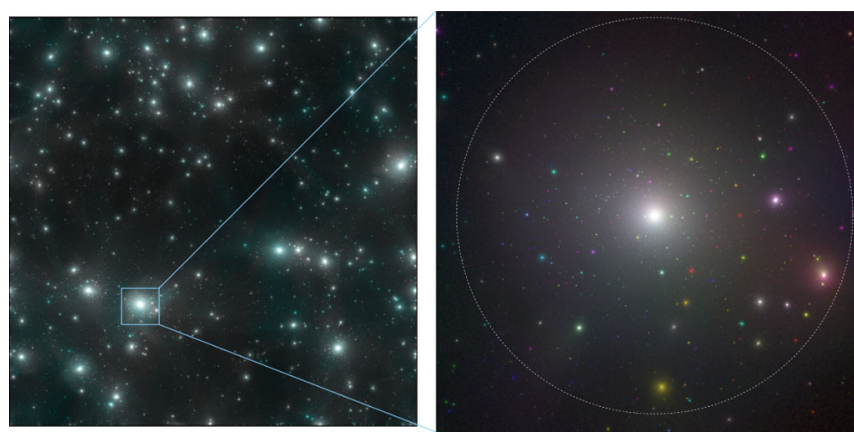

FIG. 1. Left: projected axion density of the full simulation box at $z=99$. Right: an enlargement of the largest $\mathrm{MCH}$, where the dashed circle indicates the sphere with density $\rho=200 \rho_{m, 0}$. The sub-MCs are colored according to their orbital velocity.

We follow the gravitational evolution of the system with the GADGET-3 code to a final redshift determined by the time when perturbations on the scale of the computational volume become nonlinear (see SM [26] for details). A visualization of the full simulation box at the final redshift, $z_{f}=99$, is shown in Fig. 1. An enlargement of the largest halo reveals its rich substructure.

MCHs are defined as clusters of gravitationally bound particles in close analogy with DM halos in simulations of structure formation. We identify and characterize them by their virial masses and radii using the SUBFIND halo finder [37]. At $z_{f}=99$, the masses and radii span the ranges $2.5 \times$ $10^{-16} \sim 3.0 \times 10^{-9} M_{\odot}$ and $0.4 \sim 92.0$ A.U., respectively.

Minicluster halo mass function.-The MC-HMF is the comoving number density of gravitationally bound MCHs per logarithmic mass interval as a function of $\mathrm{MCH}$ mass. It provides a quantitative picture of the dynamics of $\mathrm{MCH}$ formation.
The MC-HMF computed from our simulation for different redshifts is shown in Fig. 2. At early times $\left(z \gg z_{\text {eq }}\right.$, left panel), the MC-HMF grows quickly. It is dominated at first by halos near the low-mass resolution cutoff $\sim 10^{-15} M_{\odot}$ and develops a pronounced peak at $M_{\mathrm{mc}} \sim 10^{-13} M_{\odot}$ by $z \simeq 4 \times 10^{4}$. This rapid growth can be understood as the collapse of the density fluctuations that are deeply nonlinear at high $z$. Thus, we can identify the peak as due to the largest nonlinear fluctuations, which should be the "canonical" MCs. The abundance of low-mass MCs is the result of the small density seeds found in [22] when simulating axions with strings. The overall amplitude of the MC-HMF rises until matter-radiation equality, flattening out the peak at $M_{\mathrm{mc}}$ while extending toward higher masses.

By the time of equality $\left(z \simeq z_{\text {eq }}\right)$, the MC-HMF has developed into a power law with a slope of $\alpha \simeq-0.7$ and an exponential cutoff at $\sim 10^{-11} M_{\odot}$, corresponding to the largest canonical MCs, which typically had only $\mathcal{O}(1)$ initial overdensities [22].

During the postequality evolution $\left(z \ll z_{\text {eq }}\right.$, right panel in Fig. 2) the high-mass cutoff continues to grow at the expense of the total amplitude, which smoothly declines in time. Fitting the MC-HMF to a power law times a highmass cutoff still prefers the same overall slope $\alpha \simeq-0.7$. However, the fluctuations that collapse after $z_{\text {eq }}$ are already small (linear) and the semianalytic Press-Schechter method predicts a MC-HMF $d n / d \log M \propto M^{-1 / 2}[13,38]$, which is also compatible with the high-mass data. Indeed, a double power-law fit with cutoff provides a better fit to the MCHMF in this regime. More statistics are needed to quantify it, which we leave for future work.

The late evolution is dominated by mergers with slowly diminishing accretion of unbound axions onto existing MCHs. This is confirmed by the slow saturation of the total
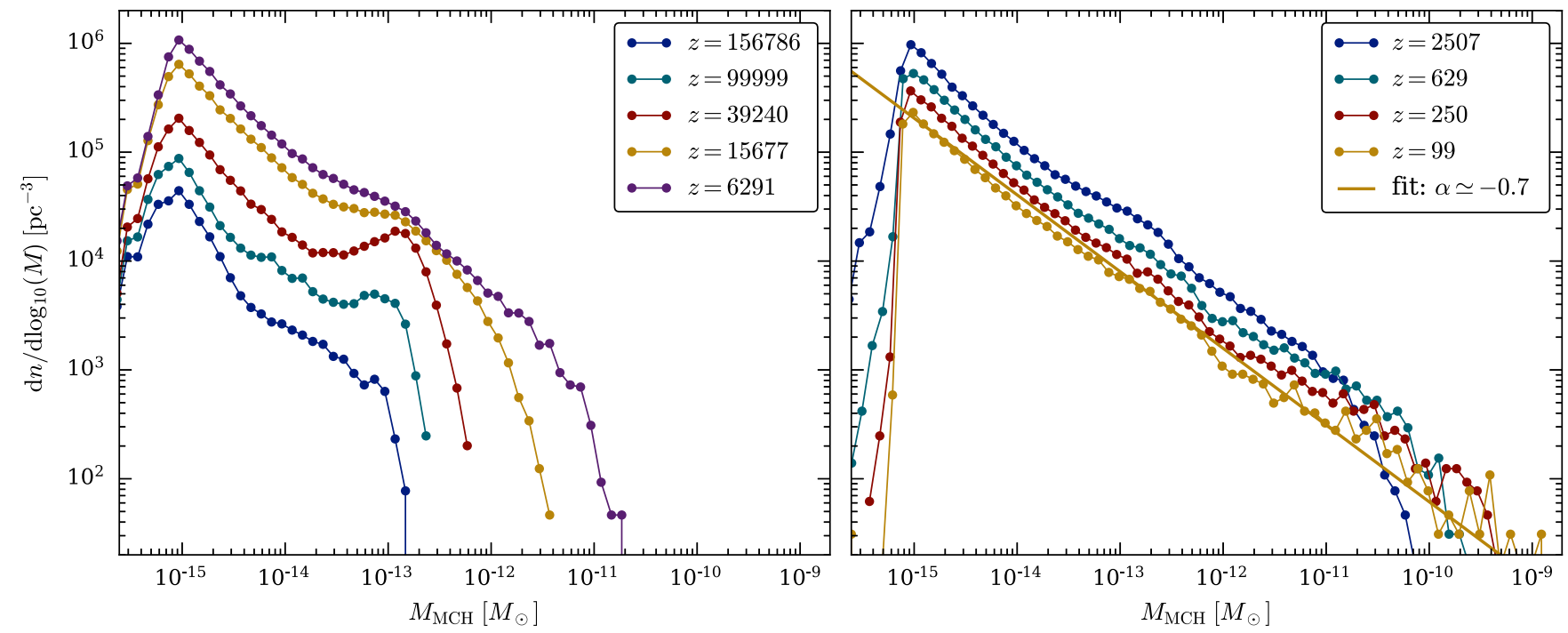

FIG. 2. MC-HMF at different redshifts $z$ separated into times before (left) and after matter-radiation equality (right). The slope of the MC-HMF at $z_{f}=99$ is $\alpha \simeq-0.7$. 


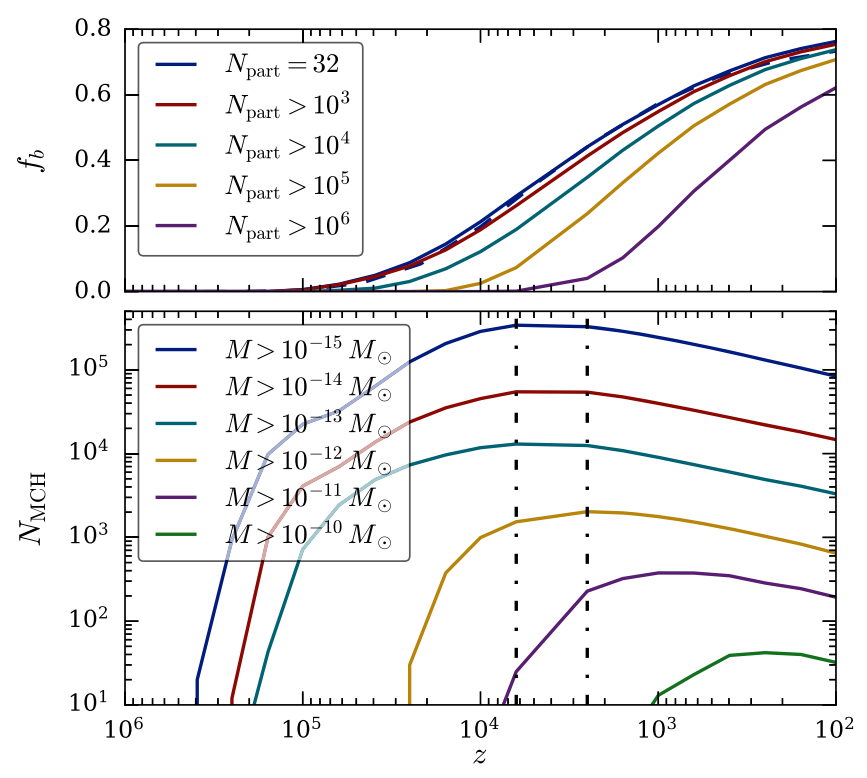

FIG. 3. Top: mass fraction $f_{b}$ of gravitationally bound axions as a function of redshift $z$ considering $\mathrm{MCHs}$ with at least $N_{\text {part }}$ particles as seen in the legend. Convergence of mass resolution for the $N_{\text {part }}=32$ case is shown by comparing simulations with $1024^{3}$ and $512^{3}$ particles (blue dotted line). Bottom: evolution of the total number of MCHs $N_{\mathrm{MCH}}$ above different mass scales as seen in the legend. The black dotted lines mark the transition from the radiation-dominated to the matter-dominated epoch.

fraction of bound axions (upper panel of Fig. 3) reaching $f_{b} \sim 0.75$ (taking into account $\mathrm{MCHs}$ with at least 32 particles) at $z_{f}=99$, and the evolution of the total number of MCHs $N_{\mathrm{MCH}}$ (lower panel of Fig. 3). Considering only MCHs with at least $10^{3}$ particles, the evolution of $f_{b}$ and the final result at $z_{f}=99$ do not change significantly. At the final redshift $60 \%$ of all axions are bound in MCHs with more than $10^{6}$ particles. Apart from this, we see that after their formation at $z \simeq 7 \times 10^{5}$ the number of MCHs grows until $z \simeq z_{\text {eq }}$. Afterwards, their number is reduced as a result of ongoing mergers. By distinguishing between $N_{\mathrm{MCH}}$ above certain mass scales we observe at which redshift $\mathrm{MCHs}$ with increasing masses emerge. Evidently, $\mathrm{MCHs}$ with masses up to $10^{-11} M_{\odot}$ begin to form before matterradiation equality while higher-mass $\mathrm{MCHs}$ arise only for $z<z_{\mathrm{eq}}$.

In order to characterize the distribution of sub-MCs within the MCHs, we compare the substructure of ten high-mass $\mathrm{MCHs}$ with ten medium-mass $\mathrm{MCHs}$ (mass samples are defined in Table I) in Fig. 4. For this, we identified all sub-MCs within the virial radius of each $\mathrm{MCH}$ and normalized the sub-MC masses to the virial mass of the corresponding parent $\mathrm{MCH}$. Figure 4 shows the relative number of sub-MCs, i.e., the number of subMCs divided by the total number $N_{\text {sub,tot }}$ of sub-MCs contained within the parent MCH. For both subsets, the slopes of the averaged sub-MC-HMFs are similar to that of
TABLE I. Selected mass samples of MCHs, their respective concentration parameter from an NFW fit, and the sensitivity of the fit to the radial fit range (details in the text).

\begin{tabular}{|c|c|c|c|c|}
\hline & $M_{\mathrm{MCH}}\left[10^{-11} M_{\odot}\right]$ & $r_{\text {vir }}$ [A.U.] & $c$ & sensitivity \\
\hline High mass & $26-300$ & $40.8-92.0$ & 160 & $3 \%$ \\
\hline Medium mass & $3.4-4.6$ & $20.7-22.8$ & 400 & $6 \%$ \\
\hline Low mass & $\sim 0.8$ & $\sim 12.7$ & 450 & $11 \%$ \\
\hline
\end{tabular}

the MC-HMF, $\alpha \simeq-0.7$. The independence of the slopes from the parent $\mathrm{MCH}$ mass agrees with previous results for subhalo mass functions in cold dark matter (CDM) simulations [39,40].

Density profiles.-We study the angular-averaged density profiles $\rho(r)$ of MCHs in the last snapshot of our simulation, $z_{f}=99$, for which we separated them into three mass samples (cf. Table I). The stacked density profiles of 20 $\mathrm{MCHs}$ in each sample, truncated at a radial distance of 4 times the numerical softening length, are plotted in Fig. 5 (upper panel) together with their best-fit Navarro-FrenkWhite (NFW) parameterizations given by [41]

$$
\rho_{\mathrm{NFW}}(r)=\frac{\rho_{0}}{r / r_{s}\left(1+r / r_{s}\right)^{2}},
$$

where $\rho_{0}$ is the characteristic density of the halo and $r_{s}$ the scale radius. For comparison, we also show the best-fit power law for the high-mass MCHs. As seen in the lower panel of Fig. 5, high-mass MCHs are in good agreement with NFW profiles across the entire radial range, and the scale radius is well resolved. The medium-mass and lowmass $\mathrm{MCHs}$, however, are slightly underdense at large radii $r \sim r_{\text {vir }} / 2$, and the scale radii from the NFW fits are close to or even below the spatial resolution limit. The deviations of the outer density profiles from the NFW fits can be possibly

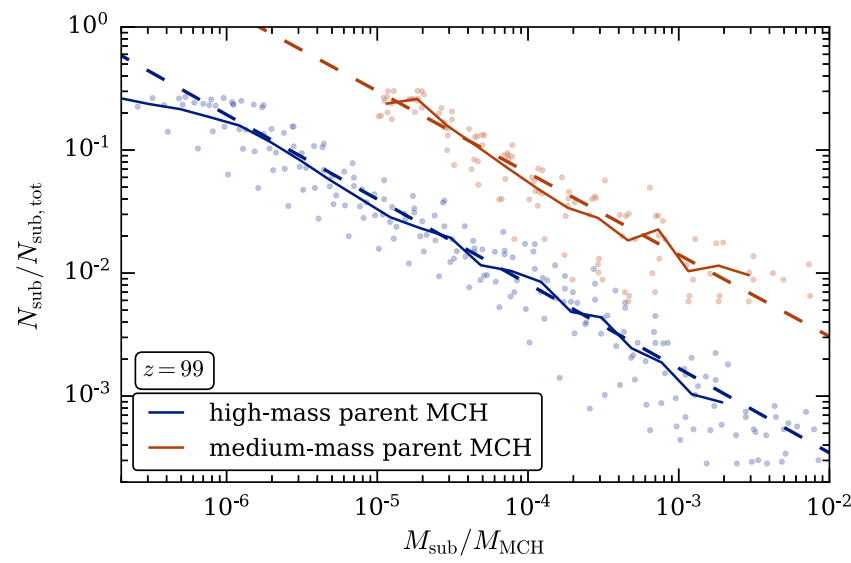

FIG. 4. Sub-MC-HMFs of ten high-mass (blue data points) and medium-mass (red data points) MCHs normalized to the virial mass of the parent $\mathrm{MCH}$ at redshift $z=99$. The solid lines represent the average of the combined data for the high-mass and low-mass MCHs, respectively. The dotted lines are power-law fits to the data, both consistent with $\alpha \simeq-0.7$. 


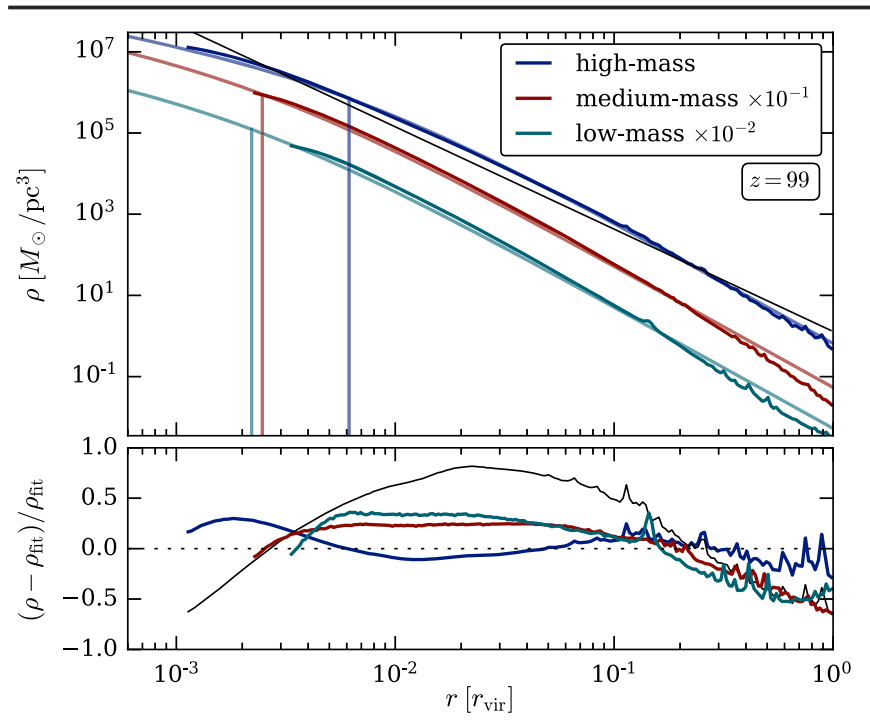

FIG. 5. Top: averaged radial density profiles of 20 miniclusters in each mass bin (dark solid lines) truncated at a radial distance of 4 times the numerical softening length. The light solid lines represent NFW fits, where vertical lines mark the corresponding scale radii. The thin black line shows the best-fit power law $\rho \sim r^{-2.52}$. Bottom: deviations from the fit shown in the upper panel.

explained by an increased mass accretion as discussed in [42].

The resulting concentration parameter, $c=r_{\mathrm{vir}} / r_{s}$, is of the order of several $10^{2}$ (cf. Table I) and increases for decreasing $\mathrm{MCH}$ masses, in agreement with $\mathrm{CDM} N$-body simulations [43]. In order to examine the stability of the fits, their radial range was reduced by $5 \%$, which varies the concentration parameter of the high-mass and mediummass sample by a few percent. The increased sensitivity for the low-mass sample is related to the fact that the scale radius is only resolved for the high-mass and medium-mass MCHs. The MCHs from the low-mass sample together with MCHs of masses down to $10^{-13} M_{\odot}$, which make up 9\% of the total number of MCHs above the low-mass resolution cutoff, have a density profile consistent with the outer $r^{-3}$ slope of NFW profiles. A verification of its convergence to Eq. (1) would require higher mass resolution and will be addressed in a follow-up publication. Nevertheless, we conclude that the density profiles at $z=99$ do not match a $\rho \sim r^{-9 / 4}$ power law predicted for spherical accretion from a homogeneous background [44]. Instead, our results are consistent with high-resolution simulations of ultracompact minihalos producing NFW density profiles for even mild deviations from spherical symmetry [45].

Studying the MCH density profiles at earlier times, we observe that they slowly converge to NFW profiles. The detailed evolution, as well as questions concerning possible differences between $\mathrm{MCHs}$ and MCs formed from mergers or monolithic collapse, are left to future work.
Discussion.-We have studied the formation of axion $\mathrm{MCs}$ and their clustering into MCHs from postinflationary symmetry breaking initial conditions. Our results are based on the highest resolution simulations performed to date, both for the initial conditions and their gravitational evolution. The main conclusions are a nearly scaleinvariant MC-HMF with slope $\alpha \simeq-0.7$, density profiles that converge toward an NFW shape for $z \ll z_{\text {eq }}$ at least for sufficiently massive $\mathrm{MCHs}$ with concentration parameters of an order of several $10^{2}$, and a final bound fraction of $f_{b} \sim 0.75$.

Of these, the bound fraction is the least robust prediction for axion $\mathrm{DM}$ at $z=0$. Improving it will require a better understanding of tidal interactions with stars in the Milky Way. For example, current estimates for tidal disruption by stellar encounters scale with the mean MC density [16,25], which is an ambiguous concept for MCHs with NFW-like density profiles. We hope that our results provide a starting point for better models, as the problem is probably intractable for full simulations.

More work is also needed to explore the morphology of $\mathrm{MCHs}$, including their mass-dependent substructure and evolution of density profiles as a function of redshift. In particular, it is plausible that features of the "original" MCs that clustered into $\mathrm{MCHs}$ remain distinguishable even at late times.

Finally, let us consider the predicted population of axion stars in the context of MCHs. Recent studies have shown that the formation of axion stars in the cores of MCHs is a firm prediction $[46,47]$. Following the relation between the mass of the axion star and the host $\mathrm{MCH}$ found in simulations [47-49], $M_{*} \sim M_{\mathrm{MCH}}^{1 / 3}$, we can expect axion stars with masses ranging from $10^{-17}-10^{-15} M_{\odot}$. Although the mass ranges of the axion stars and the smallest identified MCHs overlap, we note that our simulations are not capable of resolving them. This is because the value of the de Broglie wavelength $\lambda_{\mathrm{dB}}=(m v)^{-1}$ that determines the scale of the axion star radius does not exceed the numerical softening length across the entire mass range of the MCHs.

We thank Richard Easther, Mateja Gosenca, Shaun Hotchkiss, Doddy Marsh, Bodo Schwabe, and Jan Veltmaat for useful discussions and comments. J.R. acknowledges support from Grant Nos. PGC2018095328-B-I00 (FEDER/Agencia estatal de investigación) and FSE-DGA2017-2019-E12/7R (Gobierno de Aragón/ FEDER) and by a Mercator Fellowship with Germany“s Collaborative Research Center (SFB 1258). K. D. acknowledges support by the DFG Cluster of Excellence ORIGINS. J.C.N. acknowledges funding by a Julius von Haast Fellowship Award provided by the New Zealand Ministry of Business, Innovation, and Employment and administered by the Royal Society of New Zealand. A. V. acknowledges support by the U.S. National Science 
Foundation under Grant No. PHY14-14614. The simulations were performed at the Leibniz-Rechenzentrum under project "pr74do." B.E., J. R., and A. V. acknowledge the hospitality of the Munich Institute for Astro- and Particle Physics, funded by the Deutsche Forschungsgemeinschaft under Germany's Excellence Strategy EXC-2094390783311, during the 2020 "Axion Cosmology" workshop where some of the work in this paper was done.

*Corresponding author.

benedikt.eggemeier@phys.uni-goettingen.de

${ }^{\dagger}$ Corresponding author. jredondo@unizar.es

[1] S. Weinberg, Phys. Rev. Lett. 40, 223 (1978).

[2] F. Wilczek, Phys. Rev. Lett. 40, 279 (1978).

[3] J. E. Kim, Phys. Rev. Lett. 43, 103 (1979).

[4] M. Shifman, A. Vainshtein, and V. Zakharov, Nucl. Phys. B166, 493 (1980).

[5] M. Dine, W. Fischler, and M. Srednicki, Phys. Lett. 104B, 199 (1981).

[6] J. Preskill, M. B. Wise, and F. Wilczek, Phys. Lett.120B, 127 (1983).

[7] R. D. Peccei and H. R. Quinn, Phys. Rev. Lett. 38, 1440 (1977).

[8] R. D. Peccei and H. R. Quinn, Phys. Rev. D 16, 1791 (1977).

[9] C. Hogan and M. Rees, Phys. Lett. B 205, 228 (1988).

[10] E. W. Kolb and I. I. Tkachev, Phys. Rev. Lett. 71, 3051 (1993).

[11] E. W. Kolb and I. I. Tkachev, Phys. Rev. D 50, 769 (1994).

[12] E. W. Kolb and I. I. Tkachev, Astrophys. J. 460, L25 (1996).

[13] M. Fairbairn, D. J. E. Marsh, J. Quevillon, and S. Rozier, Phys. Rev. D 97, 083502 (2018).

[14] A. E. Nelson and H. Xiao, Phys. Rev. D 98, 063516 (2018).

[15] L. Visinelli and J. Redondo, Phys. Rev. D 101, 023008 (2020).

[16] P. Tinyakov, I. Tkachev, and K. Zioutas, J. Cosmol. Astropart. Phys. 01 (2016) 035.

[17] C. A. J. O'Hare and A. M. Green, Phys. Rev. D 95, 063017 (2017).

[18] S. Knirck, A. J. Millar, C. A. J. O'Hare, J. Redondo, and F. D. Steffen, J. Cosmol. Astropart. Phys. 11 (2018) 051.

[19] I. I. Tkachev, Pis'ma Zh. Eksp. Teor. Fiz. 101, 3 (2015) [JETP Lett. 101, 1 (2015)].

[20] M. S. Pshirkov, Int. J. Mod. Phys. D 26, 1750068 (2017).

[21] I. Tkachev, in Proceedings, 11th Patras Workshop on Axions, WIMPs and WISPs (Axion-WIMP 2015): Zaragoza, Spain, 2015 (Verlag Deutsches Elektronen-Synchrotron, DESY-PROC, 2015), pp. 173-178.

[22] A. Vaquero, J. Redondo, and J. Stadler, J. Cosmol. Astropart. Phys. 04 (2019) 012.

[23] M. Buschmann, J. W. Foster, and B. R. Safdi, Phys. Rev. Lett. 124, 161103 (2020).

[24] K. M. Zurek, C. J. Hogan, and T. R. Quinn, Phys. Rev. D 75, 043511 (2007).
[25] V. I. Dokuchaev, Yu. N. Eroshenko, and I. I. Tkachev, Zh. Eksp. Teor. Fiz. 152, 511 (2017) [J. Exp. Theor. Phys. 125, 434 (2017)].

[26] See Supplemental Material at http://link.aps.org/ supplemental/10.1103/PhysRevLett.125.041301 for details regarding the initial conditions (Sec. S1), the power spectrum (Sec. S2), a comparison to adiabatic perturbations (Sec. S3), and the performed $N$-body simulations (Sec. S4), which includes Refs. [27-36].

[27] M. Gorghetto, E. Hardy, and G. Villadoro, J. High Energy Phys. 07 (2018) 151.

[28] V. B. Klaer and G. D. Moore, J. Cosmol. Astropart. Phys. 10 (2017) 043.

[29] V. B. Klaer and G. D. Moore, J. Cosmol. Astropart. Phys. 11 (2017) 049.

[30] L. Fleury and G. D. Moore, J. Cosmol. Astropart. Phys. 01 (2016) 004.

[31] L. M. Fleury and G. D. Moore, J. Cosmol. Astropart. Phys. 05 (2016) 005.

[32] M. Kawasaki, T. Sekiguchi, M. Yamaguchi, and J. Yokoyama, Prog. Theor. Exp. Phys. 2018, 091 E01 (2018).

[33] A. Drew and E. P. S. Shellard, arXiv:1910.01718.

[34] V. Springel, Mon. Not. R. Astron. Soc. 364, 1105 (2005).

[35] C. Power, J. F. Navarro, A. Jenkins, C. S. Frenk, S. D. M. White, V. Springel, J. Stadel, and T. R. Quinn, Mon. Not. R. Astron. Soc. 338, 14 (2003).

[36] T. Zhang, S. Liao, M. Li, and L. Gao, Mon. Not. R. Astron. Soc. 487, 1227 (2019).

[37] V. Springel, S. D. M. White, G. Tormen, and G. Kauffmann, Mon. Not. R. Astron. Soc. 328, 726 (2001).

[38] J. Enander, A. Pargner, and T. Schwetz, J. Cosmol. Astropart. Phys. 12 (2017) 038.

[39] G. De Lucia, G. Kauffmann, V. Springel, S. D. M. White, B. Lanzoni, F. Stoehr, G. Tormen, and N. Yoshida, Mon. Not. R. Astron. Soc. 348, 333 (2004).

[40] K. Dolag, S. Borgani, G. Murante, and V. Springel, Mon. Not. R. Astron. Soc. 399, 497 (2009).

[41] J. F. Navarro, C. S. Frenk, and S. D. M. White, Astrophys. J. 462, 563 (1996).

[42] B. Diemer and A. V. Kravtsov, Astrophys. J. 789, 1 (2014).

[43] J. F. Navarro, E. Hayashi, C. Power, A. R. Jenkins, C. S. Frenk, S. D. M. White, V. Springel, J. Stadel, and T. R. Quinn, Mon. Not. R. Astron. Soc. 349, 1039 (2004).

[44] E. Bertschinger, Astrophys. J. Suppl. Ser. 58, 39 (1985).

[45] M. Gosenca, J. Adamek, C. T. Byrnes, and S. Hotchkiss, Phys. Rev. D 96, 123519 (2017).

[46] D. G. Levkov, A. G. Panin, and I. I. Tkachev, Phys. Rev. Lett. 121, 151301 (2018).

[47] B. Eggemeier and J. C. Niemeyer, Phys. Rev. D 100, 063528 (2019).

[48] H.-Y. Schive, M.-H. Liao, T.-P. Woo, S.-K. Wong, T. Chiueh, T. Broadhurst, and W. Y. Pauchy Hwang, Phys. Rev. Lett. 113, 261302 (2014).

[49] J. Veltmaat, J. C. Niemeyer, and B. Schwabe, Phys. Rev. D 98, 043509 (2018). 